\title{
Applying ab initio Calculations to Theoretically Investigate the Electronic Structure of the Calcium Sulfide Molecule CaS
}

\author{
Hanan Hijazi ${ }^{1} \&$ Mahmoud Korek $^{1}$ \\ ${ }^{1}$ Faculty of Science, Beirut Arab University, P. O. Box 11-5020 Riad El Solh, Beirut 1107 2809, Lebanon \\ Correspondence: Mahmoud Korek, Faculty of science, Beirut Arab University, P.O.Box 11-5020, Beirut Lebanon. \\ E-mail: mahmoud.korek@bau.edu.lb, fkorek@yahoo.com
}

Received: Sep. 11, 2018

Accepted: Sep. 25, 2018

Online Published: December 5, 2018

doi:10.5539/mas.v13n1p1

URL: https://doi.org/10.5539/mas.v13n1p1

\begin{abstract}
Using the graphical user interface GABEDIT and the computational chemistry software MOLPRO, the ab initio calculation method has been applied to explore 25 low-lying singlet and triplet electronic states, including the $\mathrm{X}^{1} \Sigma^{+}$ ground state, of the calcium sulfide molecule $\mathrm{CaS}$ in the ${ }^{2 \mathrm{~s}+1} \Lambda^{ \pm}$representation. The multi-reference configuration interaction with Davidson correction (MRCI+Q) and the complete active space self-consistent field (CASSCF) calculations were performed to obtain the potential energy curves in terms of the internuclear distance R. The permanent dipole moments $\mu_{\mathrm{e}}$ of these low-lying electronic states of $\mathrm{CaS}$ have been investigated, in addition to the corresponding spectroscopic constants (including the electronic energy with respect to the ground state $T_{e}$, the equilibrium internuclear distance $R_{e}$, the harmonic vibrational frequency $\omega_{e}$, the rotational constant $B_{e}$, and the equilibrium dissociation energy $\mathrm{D}_{\mathrm{e}}$ ). In the present work, 19 new singlet and triplet $\mathrm{CaS}$ electronic states were investigated for the first time. In addition, it is noticeable that the current results and those already available in literature are in good agreement.
\end{abstract}

Keywords: calcium sulfide, electronic structure, potential energy curves, permanent dipole moment curves, spectroscopic constants, ab initio calculation, computational chemistry

\section{Introduction}

The properties of metal sulfides have been of great interest among scientists in the domain of chemistry and other related fields due to their wide range of applications that are based on their unique metal-sulfur bond nature (Halfen, Apponi, Thompsen, \& Ziurys, 2001). For instance, in reactions involving proteins in biochemistry, metal sulfides play the role of sulfur-donating ligands (Holm, Kennepohl \& Solomon, 1996) (Halfen, Apponi, Thompsen, \& Ziurys, 2001) (Janczyk \& Ziurys, 2006). These compounds are also important in high temperature chemistry, chemical vapor deposition and corrosion processes (Bradley, 1989) (Halfen, Apponi, Thompsen, \& Ziurys, 2001). Besides, some applications in astrophysics are related to alkaline-earth metal sulfides in specific (Takano, Yamamoto, \& Saito, 1989) (Janczyk \& Ziurys, 2006).

Not many studies have been performed on the calcium sulfide molecule and they have been restricted to very few low-lying electronic states even though several theoretical and experimental investigations were done on various other alkaline-earth metal sulfides (Huber \& Herzberg, 1979) (Hüttner, 2012). Experimentally, Blues and Barrow were the first to study the absorption of $\mathrm{CaS}$ at around $1900^{\circ} \mathrm{C}$ in the year 1968 , where only 2 states were obtained and the ground state was specified as $\mathrm{X}^{1} \Sigma^{+}$(Blues \& Barrow, 1969). In 1987, a theoretical study (based on ab initio calculations) was conducted, by Partridge et al., on alkaline-earth mono-sulfides, including calcium mono-sulfide, in which 5 computational methods were used on the molecule $\mathrm{CaS}$ and 3 of its electronic states were reported (Partridge, Langhoff, \& Bauschlicher, 1988). More experimental studies were done on CaS between the years 1989 and 2002. Takano et al. used a source-modulated microwave spectrometer to observe rotational transitions of this molecule (in the $\mathrm{X}^{1} \Sigma^{+}$electronic state) in the frequency region 200 to $300 \mathrm{GHz}$ (Takano, Yamamoto, \& Saito, 1989). Jarman et al. obtained 3 electronic states (2 singlet and 1 triplet $\Pi$ states) in the blue region of calcium sulfide's spectrum (Jarman, Hailey, \& Bernath, 1992). Andersson and Davis studied the singlet $X^{1} \Sigma^{+}$and $(2)^{1} \Sigma^{+}$states of $\mathrm{CaS}$ by a Fourier Transform Spectrophotometer and calculated the corresponding principal molecular constants (Andersson \& Davis, 2001). The same 2 states were also inspected again using high-resolution laser excitation spectroscopy by Melville \& Coxon (Melville \& Coxon, 2002).

In the current study, the ab initio method was used to investigate several low-lying electronic states of calcium 
sulfide, in the ${ }^{2 s+1} \Lambda^{ \pm}$representation, by means of the complete active space self-consistent field (CASSCF) and the multi-reference configuration interaction (MRCI) with Davidson correction $(+Q)$ computational approaches. In this work, we present 25 electronic states of $\mathrm{CaS}$, out of which 19 new low-lying states are examined for the first time. Their corresponding PECs (potential energy curves) and PDMCs (permanent dipole moment curves) are demonstrated. Moreover, we were able to calculate, for 18 electronic states, the spectroscopic constants $T_{e}$ (the electronic energy with respect to the ground state), $R_{\mathrm{e}}$ (the equilibrium internuclear distance), $\omega_{\mathrm{e}}$ (the harmonic vibrational frequency), $\mathrm{B}_{\mathrm{e}}$ (the rotational constant) and $\mathrm{D}_{\mathrm{e}}$ (the equilibrium dissociation energy).

\section{Computational Approach}

The computational chemistry software MOLPRO (Werner, Knowles, Knizia, Manby, \& Schütz, 2011) has been used, in the present work, with the aid of the graphical user interface GABEDIT (Allouche, 2010) to perform $a b$ initio calculations to the diatomic molecule calcium sulfide. The state averaged CASSCF (complete active space self-consistent field) and the MRDSCI+Q (multi-reference doubly and singly configuration interaction with Davidson correction) methods have been applied to study the low-lying singlet and triplet electronic states of the $\mathrm{CaS}$ compound. The neutral calcium atom Ca with 20 electrons (10 core and 10 valence electrons), has been treated, for $s, p$ and $d$ functions $(6 s, 6 p, 5 d) /[4 s, 4 p, 2 d]$, with the Wood-Boring (WB) energy-consistent effective core potential ECP10MWB basis set described by Kaupp et al. (Kaupp, Schleyer, Stoll \& Preuss, 1991) that represents a quasi-relativistic theoretical level of reference. As for the sulfur atom $S$ of 16 electrons (16 valence electrons), in its neutral state, it has been treated with the correlation consistent polarized valence quintuple-zeta cc-pV5Z basis set given by Woon and Dunning (Woon \& Dunning, 1993) for the $s$ and $p$ functions $(20 s, 12 p, 4 d, 3 f, 2 g, 1 h) /[7 s$, $6 p, 4 d, 3 f, 2 g, 1 h] .10$ electrons (the 10 electrons of Ca), out of the 36 electrons of calcium sulfide, are kept in the core of the molecule while the other 26 valence electrons ( 10 electrons from $\mathrm{Ca}$ and 16 electrons from $\mathrm{S}$ ) are considered outside its core. The wave functions of these 26 valence electrons are defined by 24 active molecular orbitals that are formed on $3 s, 3 p, 3 d, 4 s, 4 p$ and $5 s$ of $\mathrm{Ca}$ and $1 s, 2 s, 2 p, 3 s, 3 p$ and $4 s$ of $\mathrm{S}$. During calculation, 20 electrons are frozen, by keeping them doubly occupied, in the $3 s$ and $3 p$ orbitals of calcium and the $1 s, 2 s, 2 p$, and $3 s$ orbitals of sulfur. Therefore, only the other 6 valence electrons are explicitly treated later. The active space orbitals, in the $\mathrm{C}_{2 \mathrm{v}}$ point group symmetry, are distributed into the irreducible representations $\mathrm{a}_{1}, \mathrm{~b}_{1}, \mathrm{~b}_{2}$ and $\mathrm{a}_{2}$ as $6 \mathrm{a}_{1}$, $2 \mathrm{~b}_{1}, 2 \mathrm{~b}_{2}$ and $0 \mathrm{a}_{2}$, denoted by $[6,2,2,0]$. These molecular orbitals, contained in the active space, are at least $6 \sigma(\mathrm{Ca}$ : $\left.3 d_{0}, 4 s, 4 p_{0}, 5 s ; \mathrm{S}: 3 p_{0}, 4 s\right), 3 \pi\left(\mathrm{Ca}: 3 d_{ \pm l}, 4 p_{ \pm 1} ; \mathrm{S}: 3 p_{ \pm 1}\right)$ and $1 \delta\left(\mathrm{Ca}: 3 d_{ \pm 2}\right)$.

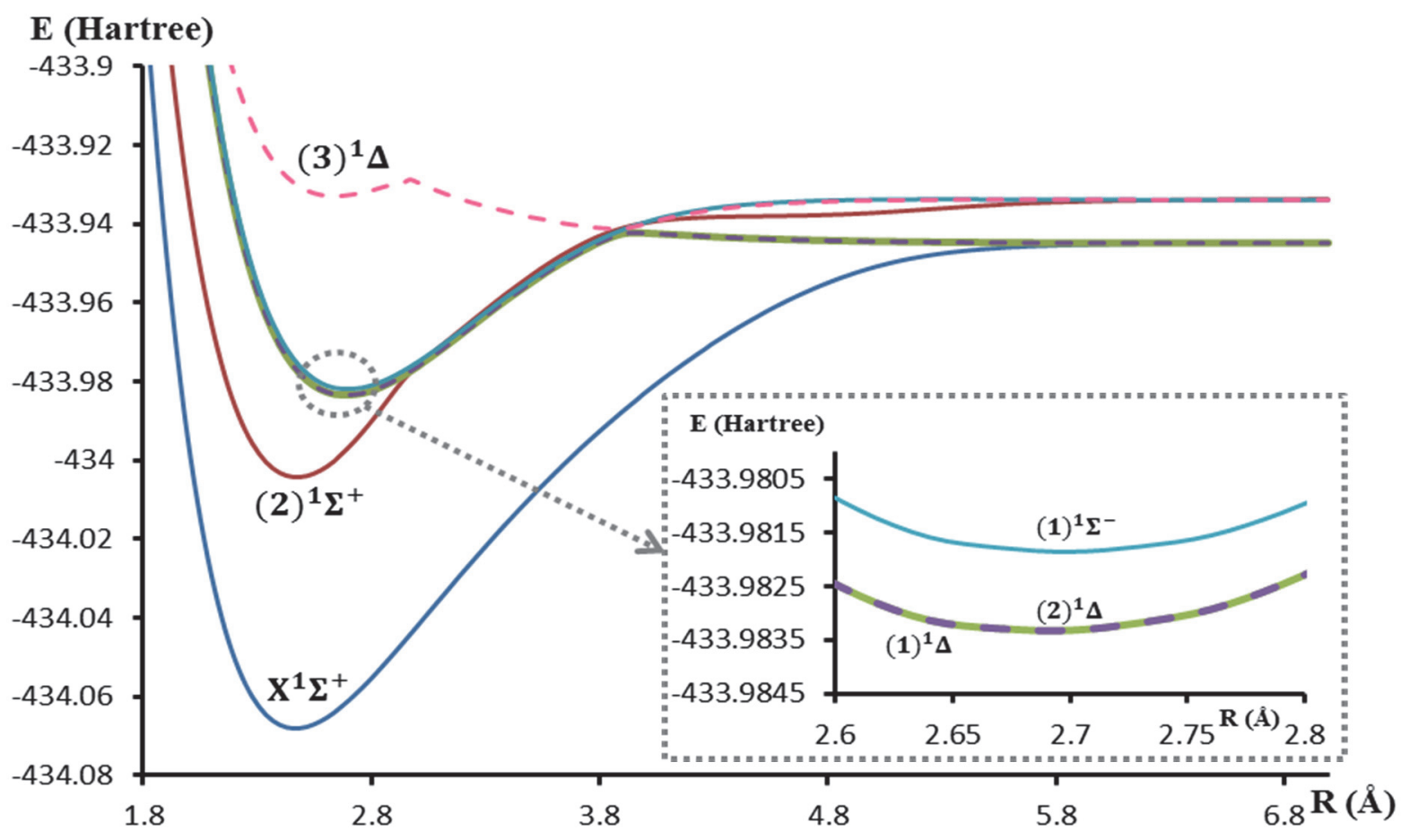

Figure 1. PECs of the singlet $\Sigma$ and $\Delta$ electronic states of CaS 


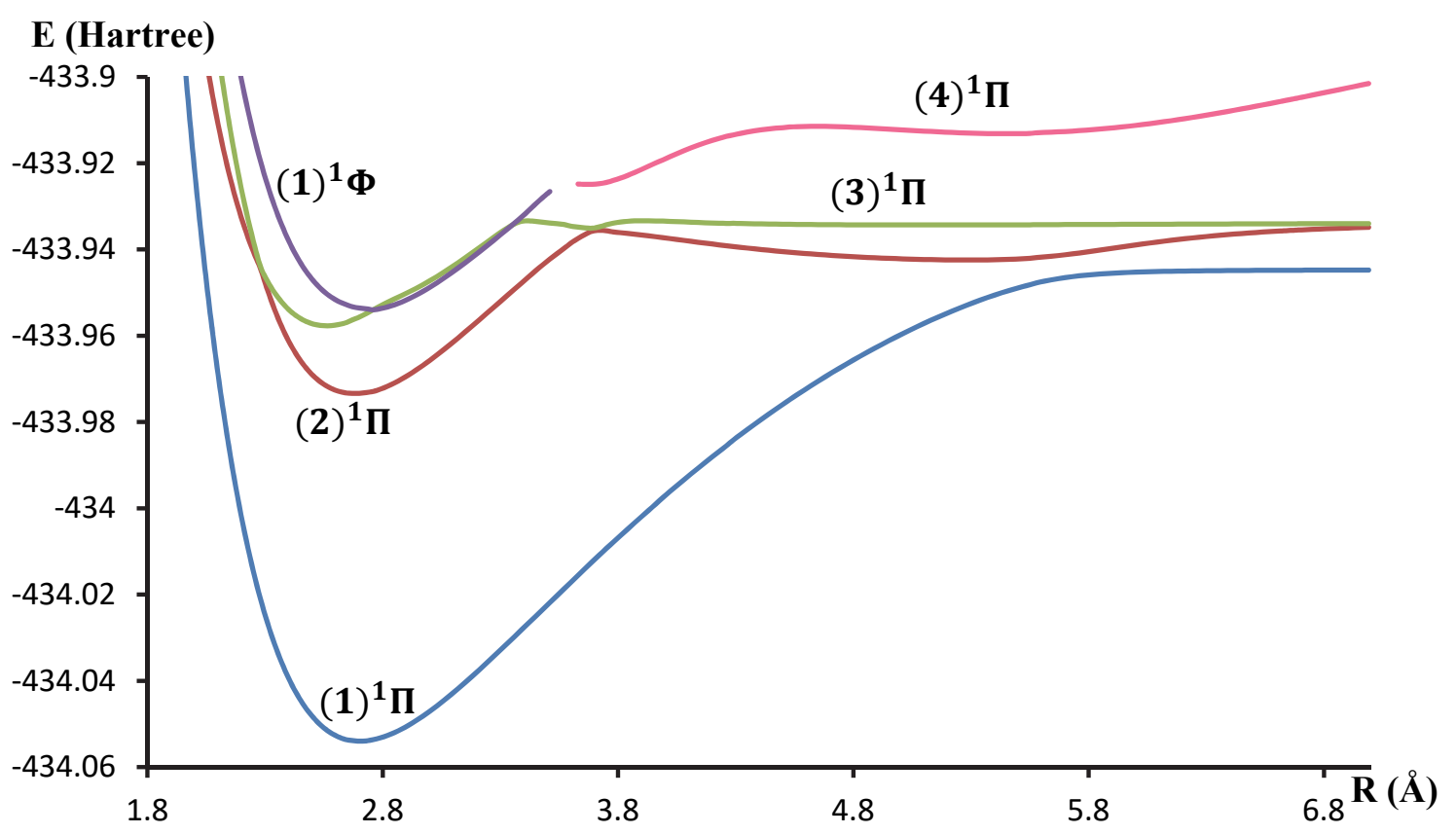

Figure 2. PECs of the singlet $\Pi$ and $\Phi$ electronic states of CaS

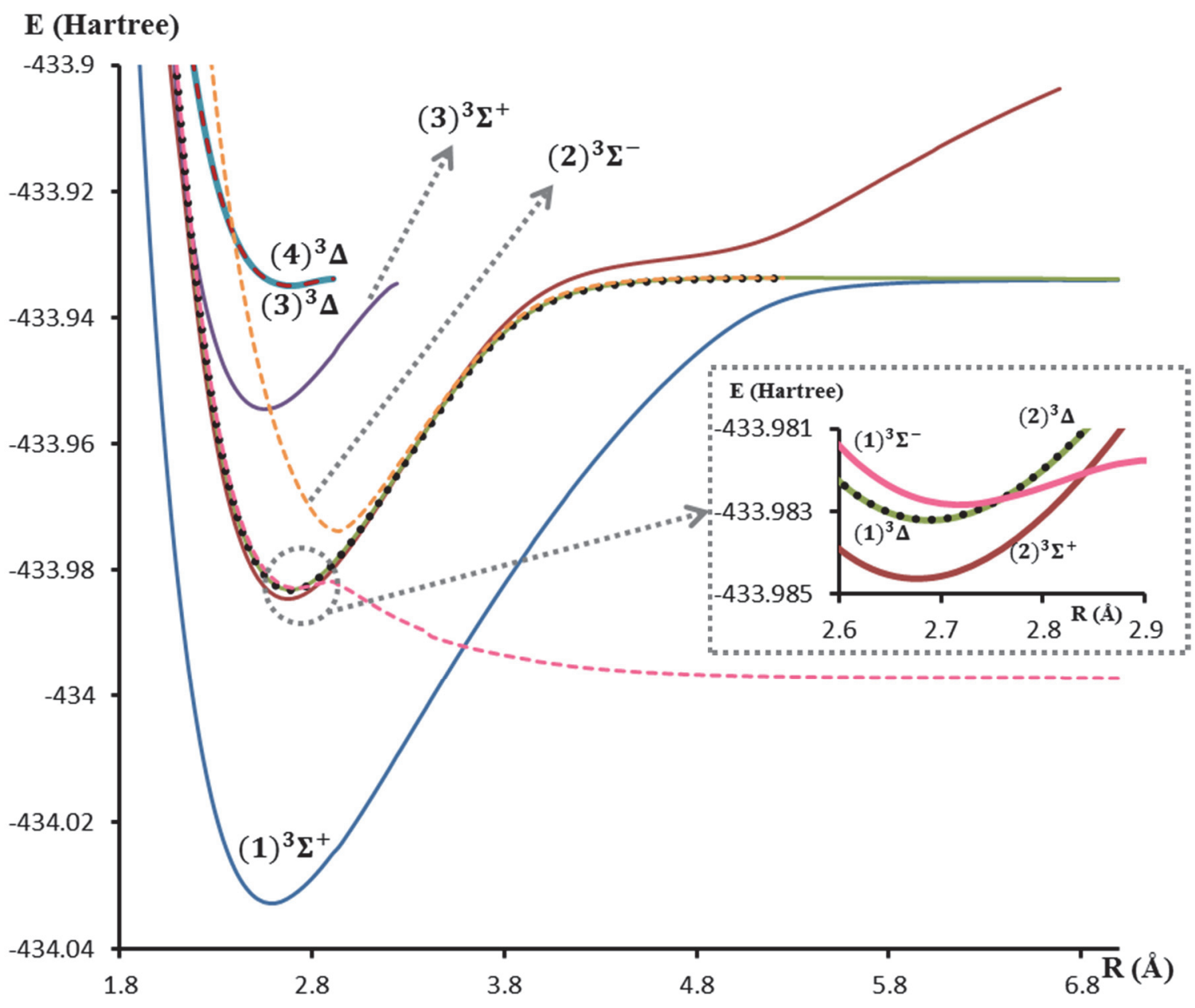

Figure 3. PECs of the triplet $\Sigma$ and $\Delta$ electronic states of CaS 


\section{Results and Discussion}

\subsection{Potential Energy Curves}

As a function of the internuclear distance R, the potential energy curves (PECs) have been computed and demonstrated for 25 low-lying singlet and triplet molecular electronic states of calcium sulfide, in the ${ }^{2 s^{+}+1} \Lambda^{ \pm}$ representation (Figs. 1-4). This PEC calculation has been applied, in the range $1.8 \AA \leq \mathrm{R} \leq 6.99 \AA$, to 174 internuclear distances.

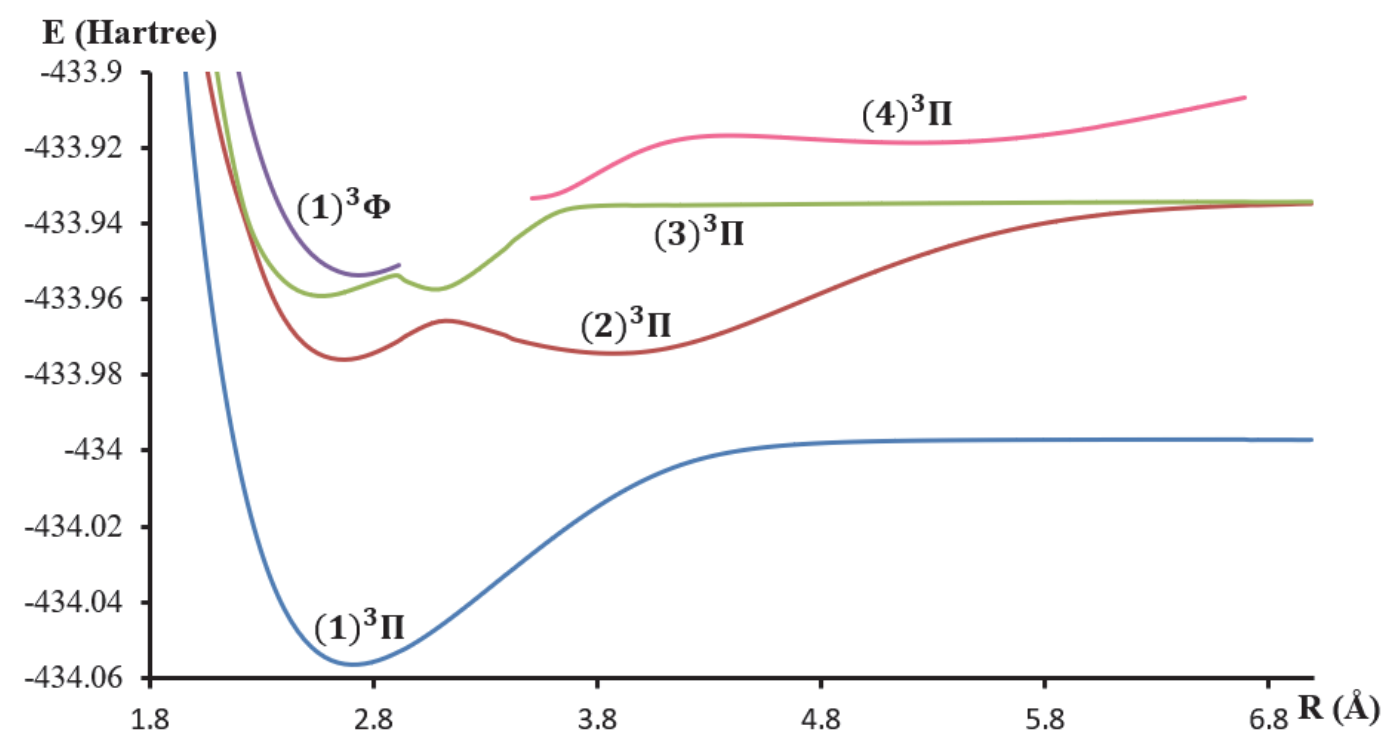

Figure 4. PECs of the triplet $\Pi$ and $\Phi$ electronic states of $\mathrm{CaS}$

In Figures 1-4, various locations of crossing and avoided-crossing, in the PECs, can be spotted between the electronic states of CaS. Sites of crossing between the PECs of the electronic states are noticed to occur as follows: $(2)^{1} \Sigma^{+}$with (1) $)^{1} \Delta$ at $\mathrm{R}=3 \AA$, (1) $)^{1} \Sigma^{-}$with $(3)^{1} \Delta$ at $\mathrm{R}=3.93 \AA$, (3) ${ }^{1} \Pi$ with $(1)^{1} \Phi$ at $\mathrm{R}=2.76 \AA$ and $\mathrm{R}=3.36 \AA$, (3) ${ }^{3} \Sigma^{+}$with $(1)^{3} \Delta$ at $\mathrm{R}=2.16 \AA$, (2) ${ }^{3} \Sigma^{+}$with $(1)^{3} \Delta$ at $\mathrm{R}=3.33 \AA$, $(1)^{3} \Sigma^{-}$with $(2)^{3} \Delta$ at $\mathrm{R}=2.76 \AA$ and $(2)^{3} \Sigma^{-}$with $(4)^{3} \Delta$ at $\mathrm{R}=2.4 \AA$. As for the sites of avoided-crossing between the PECs of the electronic states, they appear in the following cases: $X^{1} \Sigma^{+}$with $(2)^{1} \Sigma^{+}$at $\mathrm{R}=5.1 \AA$, $(2)^{1} \Pi$ with $(3)^{1} \Pi$ at $\mathrm{R}=2.28 \AA$ and $\mathrm{R}=3.72 \AA$, (3) ${ }^{1} \Pi$ with $(4)^{1} \Pi$ at $\mathrm{R}=3.75 \AA$, (1) ${ }^{1} \Pi$ with $(2)^{1} \Pi$ at $\mathrm{R}=5.67 \AA$, (1) ${ }^{1} \Pi$ with $(4)^{1} \Pi$ at $\mathrm{R}=5.31 \AA$, (2) ${ }^{1} \Delta$ with $(3)^{1} \Delta$ at $\mathrm{R}=3.9 \AA \AA$, (1) ${ }^{3} \Sigma^{+}$with $(2)^{3} \Sigma^{+}$at $\mathrm{R}=5.07 \AA$, $(2)^{3} \Sigma^{+}$with $(3)^{3} \Sigma^{+}$at $\mathrm{R}=2.16 \AA$, (1) ${ }^{3} \Sigma^{-}$ with $(2)^{3} \Sigma^{-}$at $\mathrm{R}=2.91 \AA$, $(2)^{3} \Pi$ with $(3)^{3} \Pi$ at $\mathrm{R}=2.22 \AA$ and $\mathrm{R}=3.09 \AA$, (3) $)^{3} \Pi$ with $(4)^{3} \Pi$ at $\mathrm{R}=3.69 \AA$, $(1)^{3} \Pi$ with $(2)^{3} \Pi$ at $\mathrm{R}=4.11 \AA$ and $(2)^{3} \Pi$ with $(4)^{3} \Pi$ at $\mathrm{R}=5.43 \AA$.

\subsection{Spectroscopic Constants}

Spectroscopic constants of the CaS molecule have been calculated for 18 singlet and triplet electronic states by fitting, into a polynomial in $\mathrm{R}$, the potential energy values around the equilibrium internuclear distance. These constants include the electronic energy with respect to the ground state, the equilibrium internuclear distance, the harmonic vibrational frequency, the rotational constant and the equilibrium dissociation energy, denoted as $T_{e}, R_{e}$, $\omega_{e}, B_{e}$ and $D_{e}$ respectively (Table 1). For some electronic states, where the minima of their PECs occur at regions of crossing or avoided-crossing between states, the spectroscopic constants have not been calculated.

The results, presented in Table 1, have been compared to those available in previous theoretical and experimental studies (Blues \& Barrow, 1969) (Marcano \& Barrow, 1970) (Partridge, Langhoff, \& Bauschlicher, 1988) (Takano, Yamamoto, \& Saito, 1989) (Jarman, Hailey, \& Bernath, 1992) (Andersson \& Davis, 2001) (Melville \& Coxon, 2002). The molecular spectroscopic constants of $\mathrm{CaS}$, computed here, show good agreement with the investigations found in earlier literature, especially when linked to prior experimental outcomes. The only remarkable difference in the $T_{\mathrm{e}}$ values can be noticed with respect to the CASSCF theoretical results of Partridge et al.; this is associated with the challenging nature (regarding the process of selecting configurations) of the CASSCF method which may be viewed as a full configuration interaction calculation in a restricted configuration space. On the other hand, the MRCI method (used here) is more likely to result in better enhanced computations (Kohanoff, 2006). 
Table 1. Spectroscopic constants computed for the singlet and triplet electronic states of the CaS molecule.

\begin{tabular}{|c|c|c|c|c|c|}
\hline State & $\mathrm{T}_{\mathrm{e}}\left(\mathrm{cm}^{-1}\right)$ & $\mathrm{r}_{\mathrm{e}}(\AA)$ & $\omega_{\mathrm{e}}\left(\mathrm{cm}^{-1}\right)$ & $\mathrm{B}_{\mathrm{e}}\left(\mathrm{cm}^{-1}\right)$ & $10^{8} \mathrm{D}_{\mathrm{e}}\left(\mathrm{cm}^{-1}\right)$ \\
\hline \multirow[t]{11}{*}{$\mathrm{X}^{1} \Sigma^{+}$} & $0^{\mathrm{a}}$ & $2.469^{\mathrm{a}}$ & $381.0747^{\mathrm{a}}$ & $0.155153^{\mathrm{a}}$ & $10.27^{\mathrm{a}}$ \\
\hline & $0^{\mathrm{b}}$ & $2.3178^{\mathrm{b}}$ & $462.23^{\mathrm{b}}$ & $0.17667^{b}$ & $10.2^{\mathrm{b}}$ \\
\hline & $0^{c}$ & $2.342^{\mathrm{c}}$ & $455^{\mathrm{c}}$ & & \\
\hline & $0^{\mathrm{d}, 1}$ & $2.374^{\mathrm{d}, 1}$ & $435^{\mathrm{d}, 1}$ & & \\
\hline & $0^{\mathrm{d}, 2}$ & $2.379^{\mathrm{d}, 2}$ & $441^{\mathrm{d}, 2}$ & & \\
\hline & $0^{\mathrm{d}, 3}$ & $2.343^{\mathrm{d}, 3}$ & $442^{\mathrm{d}, 3}$ & & \\
\hline & $0^{\mathrm{e}}$ & $2.317751^{\mathrm{e}}$ & $462.3^{\mathrm{e}}$ & $0.1766756^{\mathrm{e}}$ & $10.32014^{\mathrm{e}}$ \\
\hline & $0^{\mathrm{f}}$ & & $462.22856^{\mathrm{f}}$ & $\begin{array}{l}0.176675662 \\
\mathrm{f}\end{array}$ & $10.31796^{\mathrm{f}}$ \\
\hline & $0^{\mathrm{g}, 1}$ & & $462.263^{\mathrm{g}, 1}$ & $0.176673^{\mathrm{g}, 1}$ & $10.329^{\mathrm{g}, 1}$ \\
\hline & $0^{\mathrm{g}, 2}$ & & $462.265^{\mathrm{g}, 2}$ & $0.176672^{\mathrm{g}, 2}$ & $10.324^{\mathrm{g}, 2}$ \\
\hline & $0^{\mathrm{h}}$ & $2.31770^{\mathrm{h}}$ & $462.273^{\mathrm{h}}$ & $0.176683^{\mathrm{h}}$ & \\
\hline \multirow[t]{6}{*}{$(1)^{3} \Pi$} & $2516.81^{\mathrm{a}}$ & $2.709^{\mathrm{a}}$ & $296.8640^{\mathrm{a}}$ & $0.128871^{\mathrm{a}}$ & $9.71^{\mathrm{a}}$ \\
\hline & $8658^{\mathrm{d}, 1}$ & $2.637^{\mathrm{d}, 1}$ & $326^{\mathrm{d}, 1}$ & & \\
\hline & $4415^{\mathrm{d}, 2}$ & $2.641^{\mathrm{d}, 2}$ & $323^{\mathrm{d}, 2}$ & & \\
\hline & $5389^{\mathrm{d}, 3}$ & $2.600^{\mathrm{d}, 3}$ & $338^{\mathrm{d}, 3}$ & & \\
\hline & $5554^{\mathrm{d}, 4}$ & $2.642^{\mathrm{d}, 4}$ & $321^{\mathrm{d}, 4}$ & & \\
\hline & $6528^{\mathrm{d}, 5}$ & $2.597^{\mathrm{d}, 5}$ & $327^{\mathrm{d}, 5}$ & & \\
\hline$(1)^{1} \Pi$ & $3072.38^{\mathrm{a}}$ & $2.701^{\mathrm{a}}$ & $303.0692^{\mathrm{a}}$ & $0.129663^{\mathrm{a}}$ & $9.47^{\mathrm{a}}$ \\
\hline$(1)^{3} \Sigma^{+}$ & $7707.90^{\mathrm{a}}$ & $2.591^{\mathrm{a}}$ & $317.5300^{\mathrm{a}}$ & $0.140937^{\mathrm{a}}$ & $11.10^{\mathrm{a}}$ \\
\hline \multirow[t]{5}{*}{$(2)^{1} \Sigma^{+}$} & $13945.30^{\mathrm{a}}$ & $2.477^{\mathrm{a}}$ & $382.8908^{\mathrm{a}}$ & $0.154199^{\mathrm{a}}$ & $10.01^{\mathrm{a}}$ \\
\hline & $15194.44^{b}$ & $2.3864^{\mathrm{b}}$ & $409.04^{\mathrm{b}}$ & $0.16666^{\mathrm{b}}$ & $10.8^{\mathrm{b}}$ \\
\hline & $15220.775^{\mathrm{g}, 1}$ & & $409.062^{\mathrm{g}, 1}$ & $0.166680^{\mathrm{g}, 1}$ & $11.10^{\mathrm{g}, 1}$ \\
\hline & $15220.775^{\mathrm{g}, 2}$ & & $409.064^{\mathrm{g}, 2}$ & $0.1666769^{\mathrm{g}, 2}$ & $11.08^{\mathrm{g}, 2}$ \\
\hline & ${ }_{h}^{15194.4281}$ & $2.38626^{\mathrm{h}}$ & $409.077^{\mathrm{h}}$ & $0.166676^{\mathrm{h}}$ & \\
\hline$(2)^{3} \Sigma^{+}$ & $18299.54^{\mathrm{a}}$ & $2.675^{\mathrm{a}}$ & $308.1912^{\mathrm{a}}$ & $0.132223^{\mathrm{a}}$ & $9.71^{\mathrm{a}}$ \\
\hline$(1)^{1} \Delta$ & $18574.87^{\mathrm{a}}$ & $2.687^{\mathrm{a}}$ & $305.8835^{\mathrm{a}}$ & $0.131230^{\mathrm{a}}$ & $9.03^{\mathrm{a}}$ \\
\hline$(2)^{1} \Delta$ & $18576.21^{\mathrm{a}}$ & $2.687^{\mathrm{a}}$ & $300.6138^{\mathrm{a}}$ & $0.131255^{\mathrm{a}}$ & $9.35^{\mathrm{a}}$ \\
\hline$(1)^{3} \Delta$ & $18609.96^{\mathrm{a}}$ & $2.688^{\mathrm{a}}$ & $299.8309^{\mathrm{a}}$ & $0.130914^{\mathrm{a}}$ & $9.98^{\mathrm{a}}$ \\
\hline$(1)^{1} \Sigma^{-}$ & $18900.54^{\mathrm{a}}$ & $2.694^{\mathrm{a}}$ & $299.4578^{\mathrm{a}}$ & $0.130704^{\mathrm{a}}$ & $8.94^{\mathrm{a}}$ \\
\hline$(2)^{3} \Pi$ & $20195.36^{\mathrm{a}}$ & $2.664^{\mathrm{a}}$ & $305.9822^{\mathrm{a}}$ & $0.133271^{\mathrm{a}}$ & $10.11^{\mathrm{a}}$ \\
\hline$(2)^{1} \Pi$ & $20761.92^{\mathrm{a}}$ & $2.685^{\mathrm{a}}$ & $280.4175^{a}$ & $0.131217^{\mathrm{a}}$ & $11.66^{\mathrm{a}}$ \\
\hline \multirow[t]{2}{*}{$(3)^{3} \Pi$} & $23915.30^{\mathrm{a}}$ & $2.565^{\mathrm{a}}$ & $334.6192^{\mathrm{a}}$ & $0.143807^{a}$ & $10.62^{\mathrm{a}}$ \\
\hline & $23177.7302^{\mathrm{f}}$ & & $334.0689^{f}$ & $0.1459763^{f}$ & $23.64^{\mathrm{f}}$ \\
\hline \multirow[t]{2}{*}{$(3)^{1} \Pi$} & $24212.37^{\mathrm{a}}$ & $2.558^{\mathrm{a}}$ & $330.3429^{a}$ & $0.145328^{\mathrm{a}}$ & $8.84^{\mathrm{a}}$ \\
\hline & $23231.7208^{\mathrm{f}}$ & & $335.7861^{\mathrm{f}}$ & $0.14745846^{\mathrm{f}}$ & $12.793^{\mathrm{f}}$ \\
\hline$(3)^{3} \Sigma^{+}$ & $24883.38^{\mathrm{a}}$ & $2.555^{\mathrm{a}}$ & $303.8587^{\mathrm{a}}$ & $0.144910^{\mathrm{a}}$ & $13.16^{\mathrm{a}}$ \\
\hline$(1)^{3} \Phi$ & $25112.39^{\mathrm{a}}$ & $2.732^{\mathrm{a}}$ & $315.9403^{\mathrm{a}}$ & $0.126791^{\mathrm{a}}$ & $8.05^{\mathrm{a}}$ \\
\hline$(3)^{3} \Delta$ & $29176.50^{\mathrm{a}}$ & $2.690^{\mathrm{a}}$ & $247.4218^{\mathrm{a}}$ & $0.130619^{a}$ & $15.45^{\mathrm{a}}$ \\
\hline (3) ${ }^{1} \Delta$ & $29648.01^{\mathrm{a}}$ & $2.625^{\mathrm{a}}$ & $319.5126^{\mathrm{a}}$ & $0.137125^{\mathrm{a}}$ & $11.03^{\mathrm{a}}$ \\
\hline
\end{tabular}

a Present work.

${ }^{\mathrm{b}}$ Experimental work by Blues and Barrow (Blues \& Barrow, 1969).

c Theoretical estimation by Marcano and Barrow (Marcano \& Barrow, 1970).

d Theoretical work by Partridge et al. using 5 methods (d1: CASSCF, d2: CCI, d3: CCI ( $3 s 3 p)$, d4: MRCI, d5: MRCI+Core) (Partridge, Langhoff, \& Bauschlicher, 1988).

e Experimental work by Takano et al. (Takano, Yamamoto, \& Saito, 1989).

${ }^{\mathrm{f}}$ Experimental work by Jarman et al. (Jarman, Hailey, \& Bernath, 1992).

g Experimental work by Andersson and Davis (g1: Direct Approach, g2: Term Value Method) (Andersson \& Davis, 2001).

${ }^{\mathrm{h}}$ Experimental work by Melville and Coxon (Melville \& Coxon, 2002). 


\subsection{Permanent Dipole Moment Curves}

Considering the fact that it is one of the reliable physical properties (Steimle, 2000), the permanent dipole moment $\mu_{\mathrm{e}}$ has been calculated using the computational chemistry program MOLPRO (Werner, Knowles, Knizia, Manby, \& Schütz, 2011). The calculation is done by keeping, along the positive z-axis, the sulfur atom $\mathrm{S}$ free to move while fixing the calcium atom $\mathrm{Ca}$ at the origin. Accordingly, the permanent dipole moment curves (PDMCs) have been presented, in this study, for several molecular electronic states of the $\mathrm{CaS}$ molecule (Figs. 5-8).

Generally, a sharp change in the directions of the PDMCs reveals a reverse in the polarity of the atoms. Such phenomena appear at locations of the internuclear distance (R) where, in the potential energy curves (PECs), electronic states avoid crossing. In the current calcium sulfide investigation, the R sites of avoided-crossing in our PECs are actually well reflected in the sharp variations in the directions of our PDMCs (note that at R values of avoided-crossing in PECs, the PDMCs of the corresponding states may intersect due to the sharp changes in the directions of the PDMCs). Consequently, it can be deduced that the present permanent dipole moment results are of good precision.

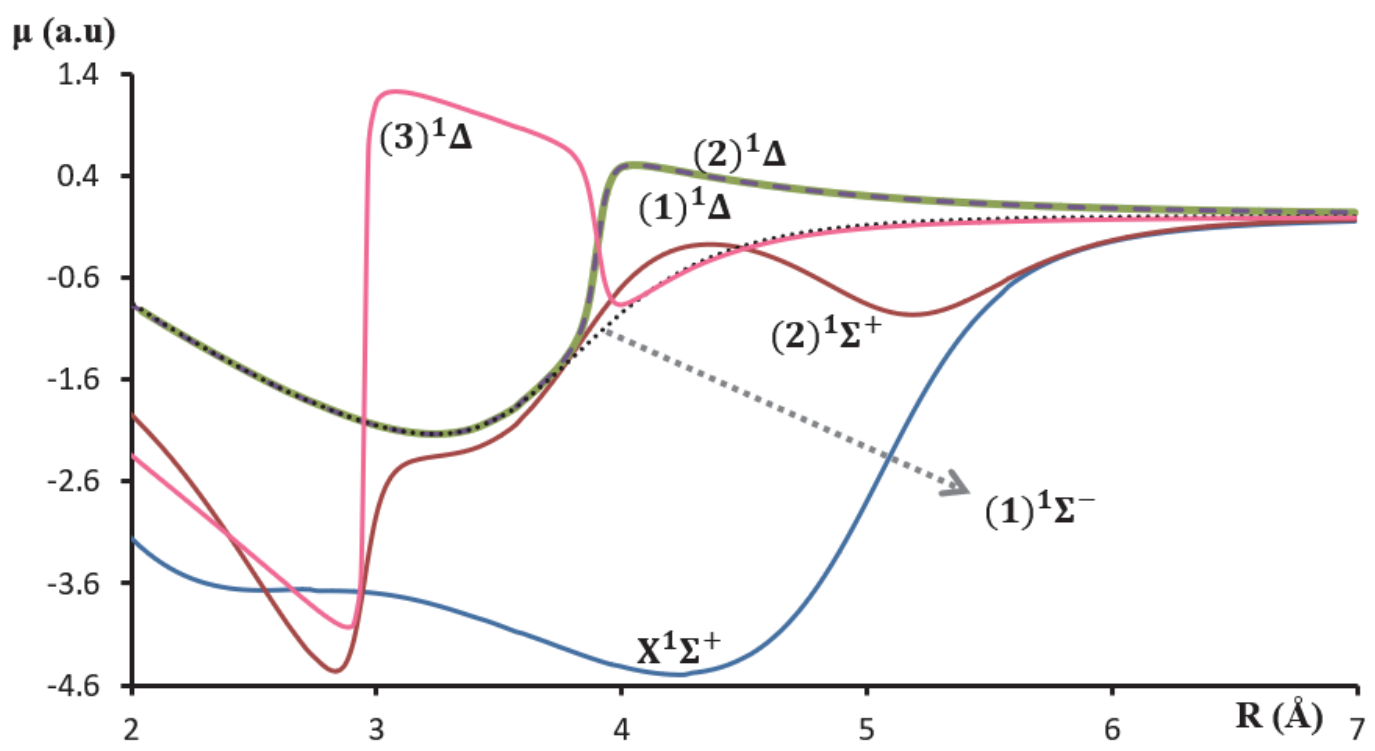

Figure 5. PDMCs of the singlet $\Sigma$ and $\Delta$ electronic states of CaS

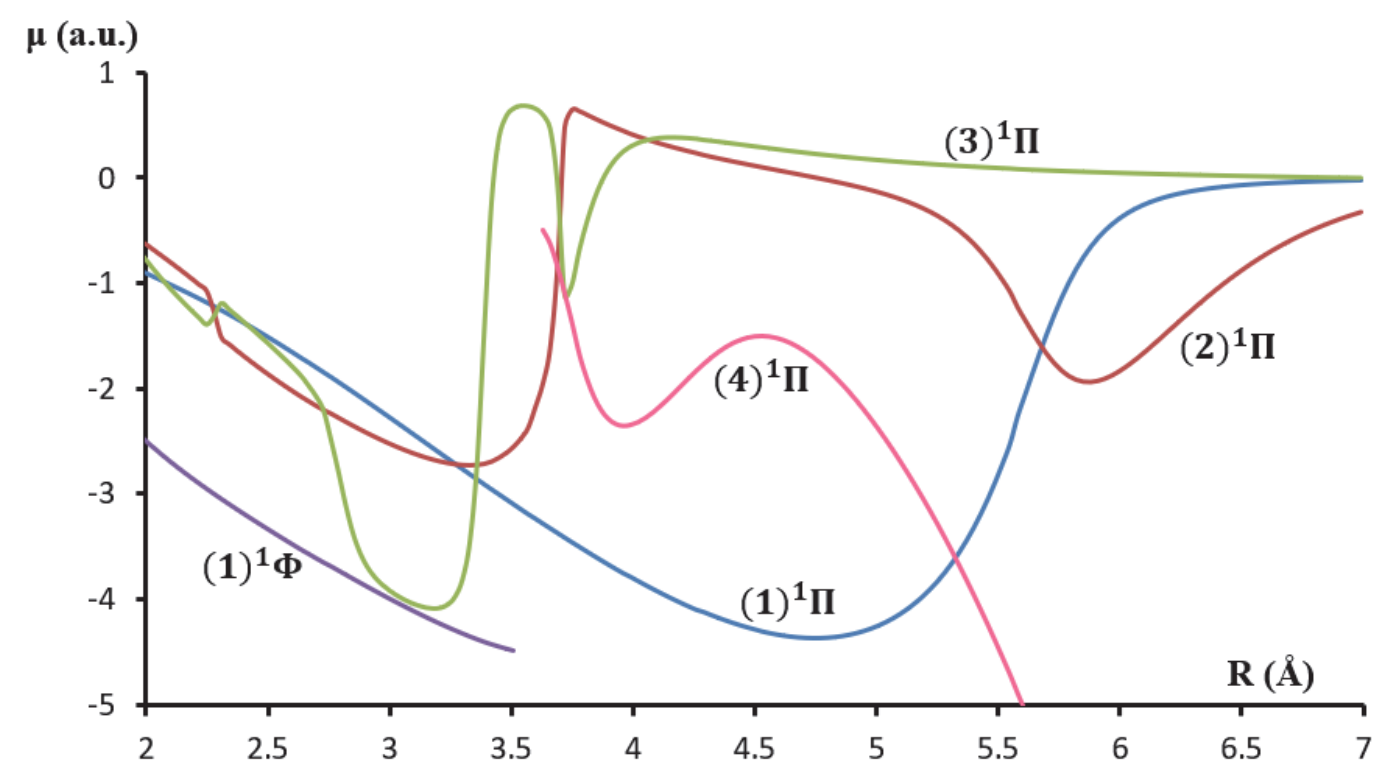

Figure 6. PDMCs of the singlet $\Pi$ and $\Phi$ electronic states of $\mathrm{CaS}$ 


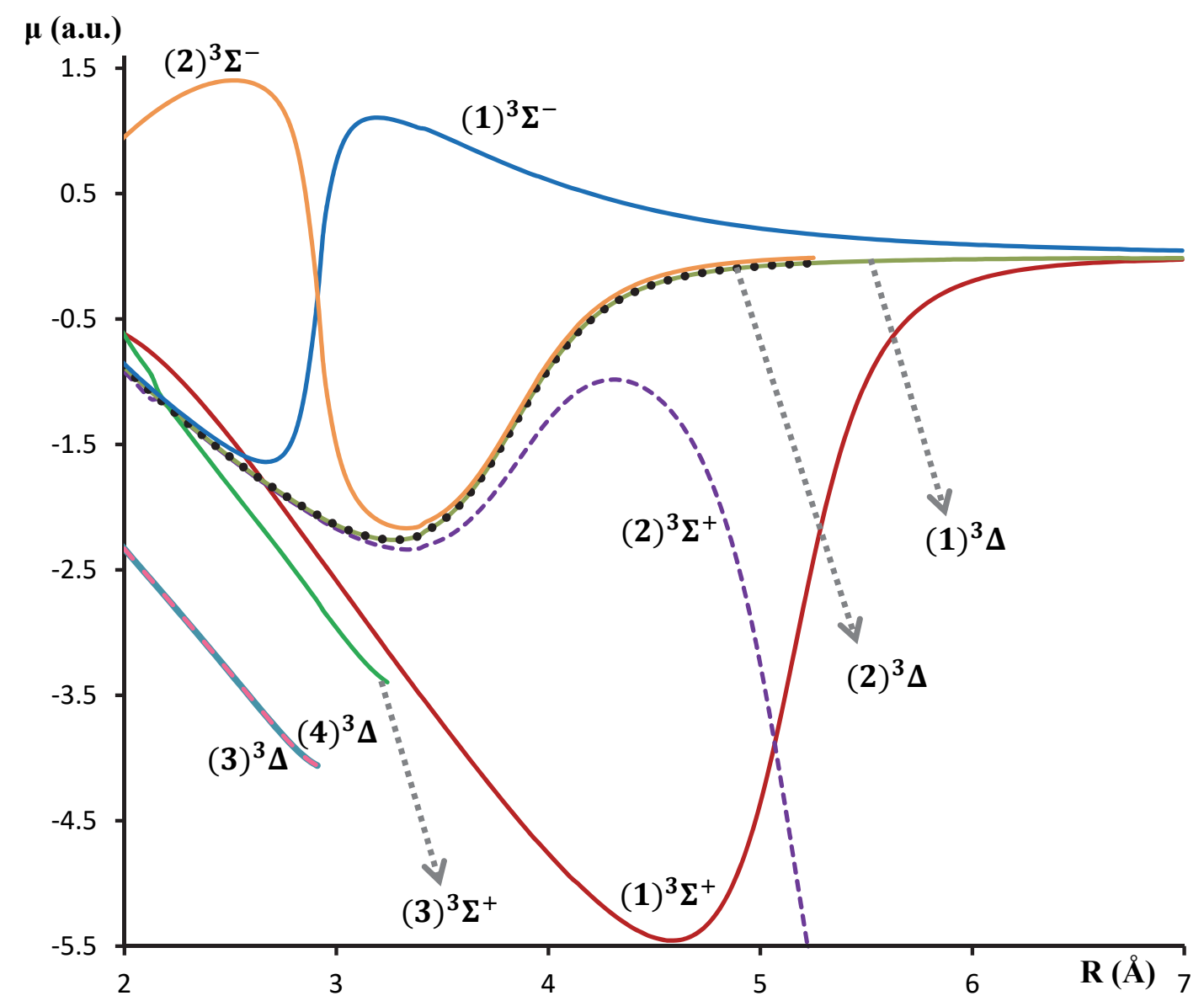

Figure 7. PDMCs of the triplet $\Sigma$ and $\Delta$ electronic states of CaS

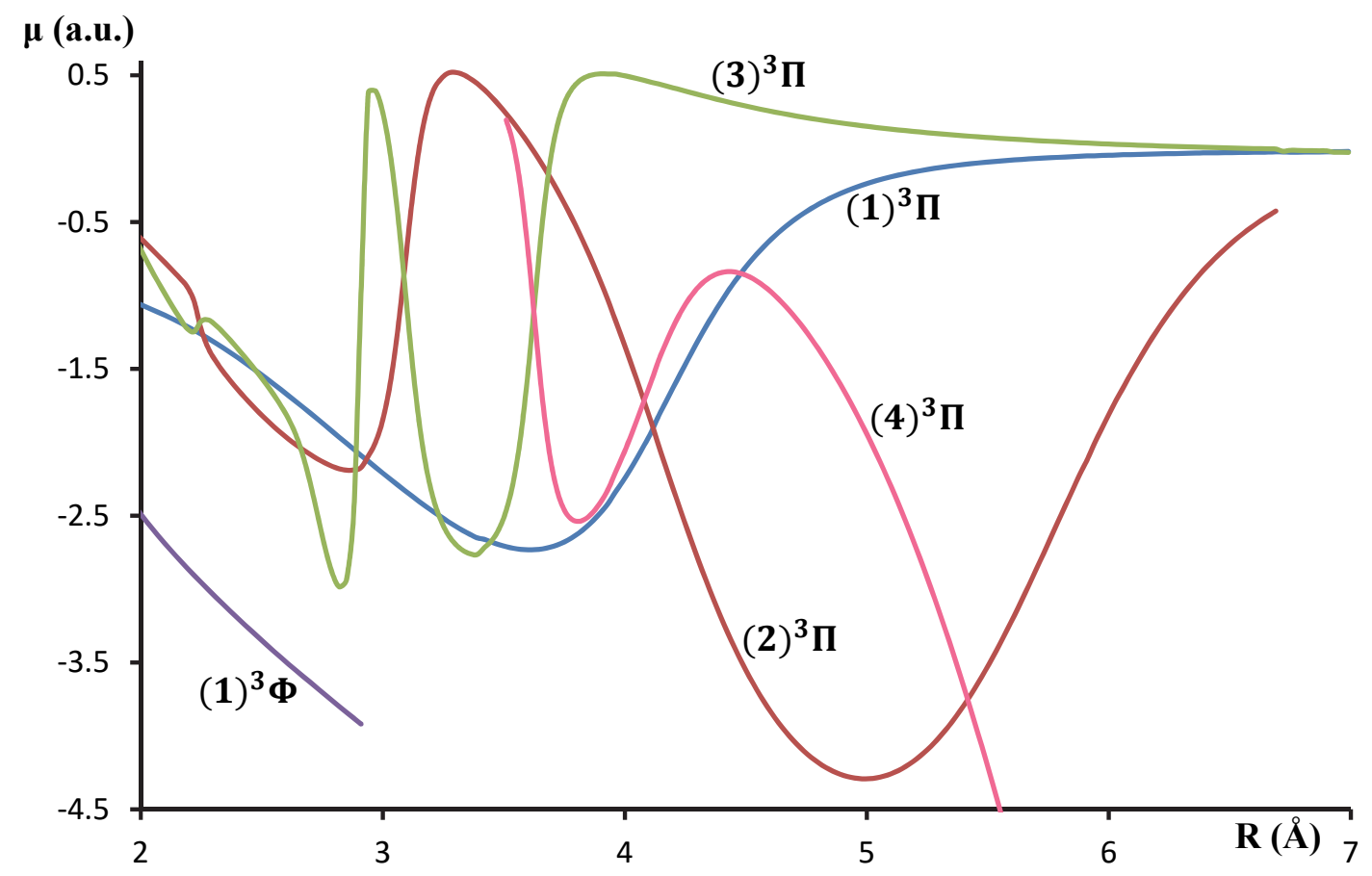

Figure 8. PDMCs of the triplet $\Pi$ and $\Phi$ electronic states of $\mathrm{CaS}$ 


\section{Conclusion}

In the current study, 25 molecular electronic states of the calcium sulfide, of which 11 are singlet and 14 are triplet, have been examined via the $a b$ initio calculation method. The CASSCF and MRCI computational methods have been applied to the calcium mono-sulfide molecule $\mathrm{CaS}$, in the ${ }^{2 \mathrm{~s}+1} \Lambda^{ \pm}$representation. Thus, the potential energy curves and the permanent dipole moment curves of this molecule have been presented. The spectroscopic constants $\left(T_{\mathrm{e}}\right.$ : the electronic energy with respect to the ground state, $\mathrm{R}_{\mathrm{e}}$ : the internuclear distance at equilibrium, $\omega_{\mathrm{e}}$ : the harmonic vibrational frequency, $\mathrm{B}_{\mathrm{e}}$ : the rotational constant and $\mathrm{D}_{\mathrm{e}}$ : the dissociation energy) have also been computed. The results of the present work are in relatively good agreement with the results of previous available literature. 19 new electronic states of $\mathrm{CaS}$ have been, to the best of our knowledge, investigated here for the first time. Hopefully, we believe our new study may provide sufficient and promising assistance in any future experimental examination applied to the calcium sulfide molecule.

\section{References}

Allouche, A. (2011). Gabedit-A graphical user interface for computational chemistry softwares. Journal of Computational Chemistry, 32(1), 174-182. https://doi.org/10.1002/jcc.21600

Andersson, N., \& Davis, S. P. (2001). Rotational Analysis of the $\mathrm{B}^{1} \Sigma^{+}-\mathrm{X}^{1} \Sigma^{+}$Transition in CaS. Physica Scripta, 64(5), 446-447. https://doi.org/10.1238/physica.regular.064a00446

Blues, R. C., \& Barrow, R. F. (1969). Rotational analysis of bands of a system $\mathrm{A}^{1} \Sigma^{+}-\mathrm{X}^{1} \Sigma^{+}$of gaseous CaS. Trans. Faraday Soc., 65(0), 646-648. https://doi.org/10.1039/tf9696500646

Halfen, D. T., Apponi, A. J., Thompsen, J. M., \& Ziurys, L. M. (2001). The pure rotational spectra of SrSH ( $\left.\tilde{X}^{2} \mathrm{~A}^{\prime}\right)$ and $\operatorname{SrS}\left(\mathrm{X}^{1} \Sigma^{+}\right)$: Further studies in alkaline-earth bonding. The Journal of Chemical Physics, 115(24), 1113111138. https://doi.org/10.1063/1.1419060

Holm, R. H., Kennepohl, P., \& Solomon, E. I. (1996). Structural and Functional Aspects of Metal Sites in Biology. Chemical Reviews, 96(7), 2239-2314. https://doi.org/10.1021/cr9500390

Huber, K. P., \& Herzberg, G. (1979). Constants of diatomic molecules. Molecular Spectra and Molecular Structure, 8-689. https://doi.org/10.1007/978-1-4757-0961-2_2

Hüttner, W. (2012). 2 Diamagnetic diatomic molecules: Introduction. Diamagnetic Diatomic Molecules. Part 1 Landolt-Börnstein - Group II Molecules and Radicals, 6-14. https://doi.org/10.1007/978-3-540-69954-5_2

Janczyk, A., \& Ziurys, L. (2006). Sub-millimeter spectroscopy of BaS $\left(\mathrm{X}^{1} \Sigma^{+}\right)$. Journal of Molecular Spectroscopy, 236(1), 11-15. https://doi.org/10.1016/j.jms.2005.11.011

Jarman, C. N., Hailey, R. A., \& Bernath, P. F. (1992). A laser study of the blue electronic transitions of CaS. The Journal of Chemical Physics, 96(8), 5571-5576. https://doi.org/10.1063/1.462698

Kaupp, M., Schleyer, P. v. R., Stoll, H., \& Preuss, H. (1991). Pseudopotential approaches to Ca, Sr, and Ba hydrides. Why are some alkaline earth MX2 compounds bent? The Journal of Chemical Physics, 94(2), 1360-1366. https://doi.org/10.1063/1.459993

Kohanoff, J. (2006). Electronic structure calculations for solids and molecules: Theory and computational methods. Cambridge: Cambridge Univ. Press.

Marcano, M., \& Barrow, R. F. (1970). Rotational analysis of a ${ }^{1} \Sigma^{+}-{ }^{1} \Sigma^{+}$system of gaseous SrS. Transactions of the Faraday Society, 66, 1917. https://doi.org/10.1039/tf9706601917

Melville, T. C., \& Coxon, J. A. (2002). High-Resolution Laser Spectroscopy of CaS: Deperturbation Analysis of the $\mathrm{A}^{1} \Sigma^{+}-\mathrm{X}^{1} \Sigma^{+}$Transition ${ }^{\dagger}$. The Journal of Physical Chemistry A, 106(36), 8271-8275. https://doi.org/10.1021/jp014299z

Partridge, H., Langhoff, S. R., \& Bauschlicher, C. W. (1988). Theoretical study of the alkali and alkaline-earth monosulfides. The Journal of Chemical Physics, 88(10), 6431-6437. https://doi.org/10.1063/1.454429

Steimle, T. C. (2000). Permanent electric dipole moments of metal containing molecules. International Reviews in Physical Chemistry, 19(3), 455-477. https://doi.org/10.1080/01442350050034199

Takano, S., Yamamoto, S., \& Saito, S. (1989). Millimeter wave spectra of MgS and CaS. Chemical Physics Letters, 159(5-6), 563-566. https://doi.org/10.1016/0009-2614(89)87533-5

Werner, H., Knowles, P. J., Knizia, G., Manby, F. R., \& Schütz, M. (2011). Molpro: A general-purpose quantum chemistry program package. Wiley Interdisciplinary Reviews: Computational Molecular Science, 2(2), 242253. https://doi.org/10.1002/wcms.82 
Woon, D. E., \& Dunning, T. H. (1993). Gaussian basis sets for use in correlated molecular calculations. III. The atoms aluminum through argon. The Journal of Chemical Physics, 98(2), 1358-1371. https://doi.org/10.1063/1.46430

\section{Copyrights}

Copyright for this article is retained by the author(s), with first publication rights granted to the journal.

This is an open-access article distributed under the terms and conditions of the Creative Commons Attribution license (http://creativecommons.org/licenses/by/4.0/). 